\title{
The dynamics of a capsule in a wall-bounded oscillating shear flow
}

LaiLai Zhu, 1,2,a) Jean Rabault, 1,3,4 and Luca Brandt ${ }^{1}$

1)Linné Flow Center and SeRC, KTH Mechanics, S-100 44 Stockholm, Sweden.

2) Laboratory of Fluid Mechanics and Instabilities, Station 9, EPFL, 1105 Lausanne, Switzerland

3) École Polytechnique, 91128 Palaiseau Cedex, France

4) Current address: Mechanics Division, Department of Mathematics, University of Oslo, 0316 Oslo, Norway

(Dated: 23 February 2018)

The motion of an initially spherical capsule in a wall-bounded oscillating shear flow is investigated via an accelerated boundary integral implementation. The neo-Hookean model is used as the constitutive law of the capsule membrane. The maximum wall-normal migration is observed when the oscillation period of the imposed shear is of the order of the relaxation time of the elastic membrane; hence, the optimal capillary number scales with the inverse of the oscillation frequency and the ratio agrees well with the theoretical prediction in the limit of high-frequency oscillation. The migration velocity decreases monotonically with the frequency of the applied shear and the capsule-wall distance. We report a significant correlation between the capsule lateral migration and the normal stress difference induced in the flow. The periodic variation of the capsule deformation is roughly in phase with that of the migration velocity and normal stress difference, with twice the frequency of the imposed shear. The maximum deformation increases linearly with the membrane elasticity before reaching a plateau at higher capillary numbers when the deformation is limited by the time over which shear is applied in the same direction and not by the membrane deformability. The maximum membrane deformation scales as the distance to the wall to the power $1 / 3$ as observed for capsules and droplets in nearwall steady shear flows.

a) Electronic mail: lailaizhu00@gmail.com 


\section{INTRODUCTION}

The dynamics of a capsule in external flows have attracted enormous interest due to their physiological and biological significance, leading to the discovery of a variety of interesting and complex phenomena. Since the observation of the tank-treading (TT) motion of a human red blood cell (RBC) in the viscometer ${ }^{1}, 2,2$, real cells and their models have been examined in shear flow, one of the simplest flows, both experimentally, theoretically and numerically in order to understand the motions of biological cells ${ }^{3-16}$. The focus was on an initially spherical capsule that undergoes shear-induced TT motion, reaching a steady ellipsoidal shape $\underline{17} \underline{18}$, when the membrane elements rotate along the stationary configuration like the caterpillar driving of a tank. The undeformed shape of biological cells is, however, seldom a sphere, rather geometrically anisotropic, the bi-concave shape of undeformed RBCs being a classic example. This non-sphericity introduces a shape memory for the capsules and tends to orient them in a preferential direction $\underline{19}$. As a consequence, the capsules tank-tread and oscillate around a preferred direction as the shear rate is above a certain value 20,21 ; this oscillatory behavior is termed as the swinging mode 22 .

Oscillating shear flows have been investigated to account for the physiological pulsation, and more complex and diverse capsule dynamics explored. Experiments 23 showed that the RBCs deform more in the retarding phase than in the accelerating phase. Cells in harmonically modulated shear were examined by theoretical approaches ${ }^{24}$ to reveal a resonance behavior; a particular combination of the oscillation frequency and phase can induce the tumbling motion of a capsule which would otherwise swing under steady shear flow. Dupire et $a l . \stackrel{25}{n}$ theoretically produced disordered motions of the RBCs under sinusoidally varying flows with physiologically relevant parameters. A similar chaotic behavior was also reproduced in the analytical investigation by Noguchi ${ }^{26}$ who found multiple limit-cycle solutions of a model RBC subject to an oscillating shear flow at high frequency; their tumbling or tank-treading motions were found at high or low shear amplitudes. The recent numerical simulations in Ref. 27 reproduce the two swinging modes of an initially oblate capsule by varying the frequency of the external shear; these authors also document the high sensitivity of the motion of the capsule to its initial orientation, indirectly confirming the chaotic motion reported in Ref. 25.

These previous studies mainly focused on the dynamics of capsules in an unbounded oscillatory shear flow. In the micro-circulation, however, cells like the RBCs move in strongly confined oscillating flows, an example being the physiological process called vasomotion. 
This corresponds to the spontaneous and rhythmic oscillations of large arteries as well as microvessels $\underline{28} \underline{-30}$. Vasomotion is independent of the common physiological pulsations induced by the heart beat, innervation or respiration $\stackrel{31}{=}$, but due to the constriction and dilation of the smooth muscle. Its possible benefits include reducing the time-averaged hydraulic resistance to the blood flow $\underline{\underline{32}}$ and the consequent enhancement of oxygen transport $\underline{33}, \underline{34}$. It is therefore important to understand the dynamics of deformable particles like biological cells in a confined oscillating flow.

In addition to the complexity introduced by the oscillation, the influence of wall confinement on the cell motion is crucial and has been, thus far, mostly investigated in steady flows where cells exhibit cross-streamline migrations in most cases. Fåhraeus and Lindqvist $\frac{35}{5}$ first unraveled the biological importance of confinement. They discovered that the RBCs tend to deform significantly and migrate towards the center of a microvessel owing to the hydrodynamic lift resulting from the interaction between the cellular deformation and the wall; this migration facilitates a lower flow resistance and a more effective mass transport. Simulations using the front-tracking method have been conducted by Doddi et al $\underline{\underline{36}}$ to investigate the cross-stream lift of a capsule towards the centerline in a Poiseuille flow; these authors observed that the migration velocity scales as the distance to the wall to the power $1 / 3$ but varies non-linearly with the capillary number. The recent numerical study by Pranay et $a l . \underline{37}$, based on an accelerated boundary integral method as that used here, investigates the lateral migration of an individual and a suspension of initially spherical capsules in wallbounded Newtonian and viscoelastic shear flows. These authors show that the migration of the capsule depends on the capillary number, and that it is attenuated by the addition of polymers. Migration has also been reported for vesicles: in an unbounded Poiseuille flow $\underline{38}$, 39 , the migration velocity depends on the flow curvature, vesicle deformability and viscosity contrast of the fluid inside and outside the vesicle; in a wall-bounded shear, the migration velocity is proportional to the wall normal component of the particle stresslet and the presence of the wall delays its transition from the tank-treading motion to trembling and tumbling $\stackrel{40}{ }$.

Recently, two groups 11,42 have numerically examined the case of a near-wall single capsule transported in a Newtonian shear flow, using a front-tracking and a boundary integral method respectively. Singh et al $\underline{\underline{41}}$ document the power-law relations between the migration velocity, capsule deformation and the capsule-wall distance, as previously done analytically for a droplet. These authors also propose a semi-analytical theory identifying two competing mechanisms influencing the lateral migration: the interfacial stresses and the viscosity ratio; 
this theory agrees well with the simulation results for large capsule-wall distances. Nix et al. $\stackrel{42}{n}$ pay more attention to the correlation between the deformation of a capsule and its migration. These authors find that an asymmetric capsule deformation reduces the migration velocity and this effect is compensated by an increase in the capsule stresslet, in turn enhancing its migration.

Very recently, Matsunaga et al. $\underline{\underline{3}}$ investigated the motion of capsule in an unbounded oscillating shear flow, observing an overshoot phenomenon when the maximum deformation of capsule can be larger than its deformation in a steady shear. In this manuscript, we extend the work in Ref. 41, Ref. 42 and Ref. 43 by considering a near-wall individual capsule in oscillating plane shear flow. We hence introduce one additional factor, i.e., the unsteadiness of the background flow that is a typical feature of biological environments. We aim to understand the interplay between the wall and the flow unsteadiness, explaining its influence on the dynamics of a capsule.

The paper is organized as follows. After the description of the problem setup, we give a brief introduction to our computational framework based on an accelerated boundary integral method suited for general geometries. We firstly report the trajectories of capsules migrating from the wall and then investigate the dependence of the lateral migration velocity on the capsule capillary number, the capsule-wall distance and the frequency of the oscillation of the background shear. We further analyze the correlation between the migration velocity and the normal stress difference that arises from the viscoelastic effects due to the deformable particles. Finally, the deformation of the capsule is examined; the phase difference between the deformation and the applied shear is measured to quantify the induced viscoelasticity. The paper ends with a summary of the main findings.

\section{PROBLEM SETUP AND NUMERICAL METHODS}

\section{A. Problem setup}

We compute the motion and deformation of a capsule subject to a wall-bounded shear flow, see the sketch in figure1. The undeformed shape of the capsule is a sphere with radius $a$ and center of mass initially located at a distance $h_{\text {ini }} / a$ above the wall. A time-periodic harmonic shear flow $\dot{\gamma}(t)=\dot{\gamma}_{\max } \cos (\omega t)$ is applied. $\dot{\gamma}_{\max }$ denotes the maximum value of the shear, and $\omega$ is the oscillation frequency. The characteristic time of the flow is thus $T=2 \pi / \omega$. In the current work, we study a capsule as a model cell and reproduce its motion 


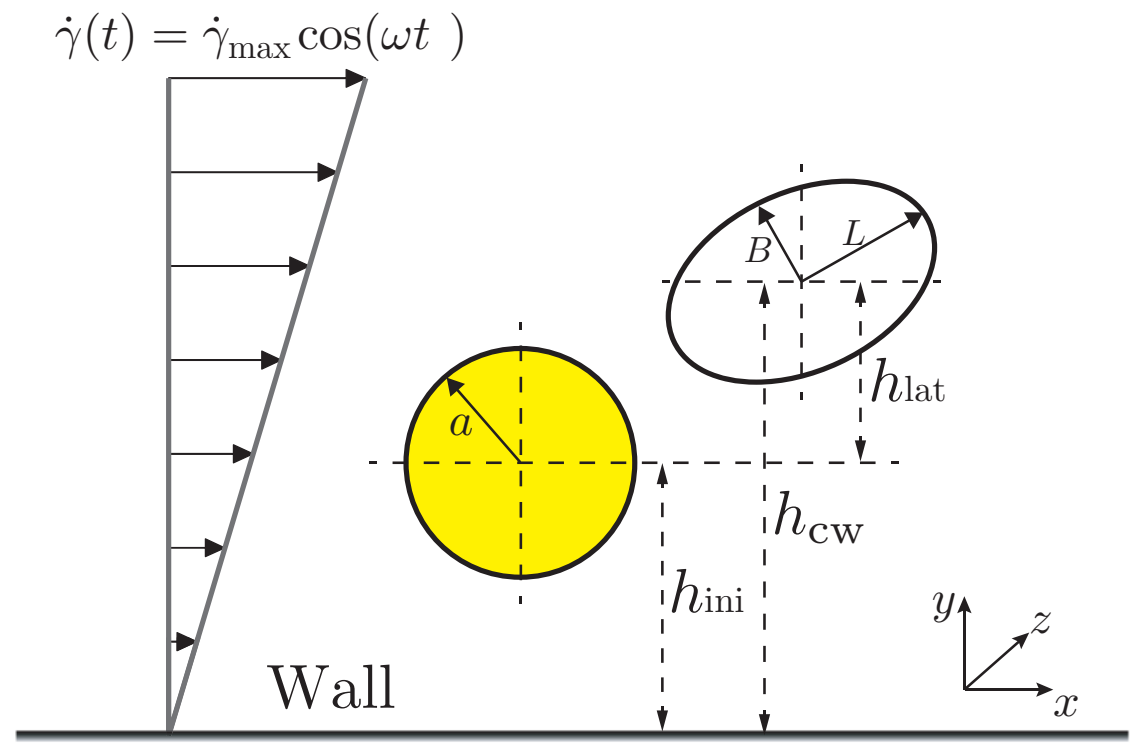

FIG. 1. (Color online) We consider a capsule whose undeformed shape is a sphere of radius $a$, subject to an oscillating wall-bounded shear flow $\dot{\gamma}(t)=\dot{\gamma}_{\max } \cos (\omega t)$. The center of mass of the capsule is initially a distance $h_{\text {ini }}$ above the wall and its lateral migration, measured from the initial position, is denoted $h_{\text {lat }}$. The distance between the capsule and the wall is thus $h_{\mathrm{cw}}=h_{\text {ini }}+h_{\text {lat }}$.

and deformation in the micro-circulation or in micro-fluidic devices. We therefore assume that the capsule is advected by a creeping flow and the Reynolds number $R e$, indicating the ratio of inertial over viscous forces, is zero.

The capsule is considered as a fluid-filled droplet enclosed by an infinitely thin elastic membrane. The fluid inside and outside the capsule has the same density $\rho$ and the same dynamic viscosity $\mu$. For real biological cells, the viscosity of the fluid inside and outside is not necessarily the same; this is not taken into account here to limit the investigated parameter space. The capsule is deformed by the fluid flow and the stress on its membrane is determined by the neo-Hookean constitutive law $\underline{\underline{44}}$. The membrane has a shear modulus $G_{\mathrm{S}}$ and zero bending stiffness. Elastic stresses develop on the surface of the capsule due to its deformation; this stress modifies the surrounding flow in return. In this fluid-structure interaction problem, the viscous stress of the flow and elastic stress of the deformable membrane compete with each other, their ratio defined as the capillary number $\mathrm{Ca}=\mu \dot{\gamma}_{\max } / G_{\mathrm{s}}$. 


\section{B. Numerical method}

The membrane of the capsule is discretized into $N$ Lagrangian points. Neglecting the inertia of the capsule, the elastic force balances the flow viscous force on its surface,

$$
\boldsymbol{\rho}^{\mathrm{e}}+\left(\sigma_{\text {out }}-\sigma_{\text {in }}\right) \cdot \mathbf{n}_{\text {out }}=0
$$

where $\boldsymbol{\rho}^{\mathrm{e}}$ is the elastic force per unit area exerted by the membrane, $\sigma_{\text {out }}, \sigma_{\text {in }}$ are the stress tensor of the flow outside and inside the capsule, and $\mathbf{n}_{\text {out }}$ is the unit outwards normal vector. Given the distribution of $\boldsymbol{\rho}^{\mathrm{e}}$ on the membrane, the flow obeys at each time the Stokes equations with $N$ point forces exerted on the fluid:

$$
\begin{aligned}
-\nabla p+\mu \boldsymbol{\nabla}^{2} \mathbf{u} & =-\sum_{i=1}^{N} \mathbf{f}_{i} \delta\left(\mathbf{x}-\mathbf{x}_{i}\right) \\
\boldsymbol{\nabla} \cdot \mathbf{u}(\mathbf{x}) & =0
\end{aligned}
$$

where $p$ and $\mathbf{u}$ are the pressure and velocity field. $\mathbf{f}_{i}$ denotes the force on the fluid at the position $\mathbf{x}_{i}$ and can be approximated by

$$
\mathbf{f}_{i}=\int_{S_{i}} \boldsymbol{\rho}_{i}^{\mathrm{e}} d S_{i},
$$

where $S_{i}$ represents the elemental patch around the $i$ th Lagrangian point. We solve the governing flow equations with a boundary integral method, accelerated by the general ge-

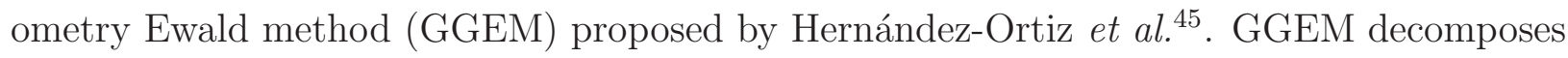
the Stokes solution into two parts: i) the short-ranged interactions computed by traditional boundary integral techniques; and ii) the long-ranged interactions handled by a mesh-based Stokes solver. In our implementation, we take the Stokes sub-solver of the open source software NEK5000 $\underline{46}$ as the mesh-based solver. We perform singular and nearly-singular integration for an accurate near-field solution, see Ref. 47. A proper treatment of the two is crucial to achieve the necessary numerical accuracy. $\underline{48}, \underline{49}$

The flow domain is infinite and bounded only by a plane wall. However, in the framework of GGEM, a computational domain of finite size is needed; here we choose $24 a(x) \times$ $24 a(y) \times 15 a(z)$. We impose periodic boundary direction in the streamwise $(x)$ and spanwise (z) direction, while keeping the bottom wall stationary and imposing at the upper boundary a time-periodic velocity in the $x$ direction. The domain is discretized by 4000 cubic spectral elements with $4 \times 4 \times 4$ Gauss-Lobatto-Legendre points and the grid is refined in the region where the capsule moves. This strategy has been used to study a capsule in 
an unbounded shear, producing results in agreement with published data obtained with a traditional boundary integral method $\underline{8}$.

Once the flow solution is available, the velocity is known at each Lagrangian point, $\mathbf{u}\left(\mathbf{x}_{i}\right)$. Due to the no-slip, non-penetrating boundary condition on the membrane of the capsule, the rate of change of the position of the $i$ th point is given by

$$
\frac{d \mathbf{x}_{i}}{d t}=\mathbf{u}\left(\mathbf{x}_{i}\right)
$$

Given the new coordinates of the $N$ Lagrangian points, we are able to calculate the elastic force per unit area on each point. The computation of the stress is based on the displacement with respect to the undeformed shape of the capsule, through the constitutive law of the membrane, the neo-Hookean model here. We use a global spectral method based on the spherical harmonics 47 to represent the surface of the capsule and to solve for the elastic force $\boldsymbol{\rho}^{\mathrm{e}}$. The advantage of this approach is twofold: i) high order spatial derivatives on the material points are computed with high accuracy, which is crucial for the calculation of the elastic force; ii) the same spectral discretization can be used for the boundary integration performed when solving the short-ranged hydrodynamic interactions. For the details of our implementation, the readers are referred to Refs. 50, 51. One of the important features is the capability of simulating deformable capsules in general geometries, as illustrated by the design of a deformability-based cell sorting device ${ }^{52}$ and a constricted micro-fluidic channel that can be used to infer the mechanical properties of cellular particles 53 .

\section{RESULTS}

\section{A. Trajectories}

We firstly look at the trajectories of the capsules. The time evolution of the center of mass and surface centroid of the capsule are depicted in the shear plane $(x-y$ plane) in figure 2 for three cases, $\left(C a, \omega / \dot{\gamma}_{\max }\right)=(0.15,1),\left(C a, \omega / \dot{\gamma}_{\max }\right)=(0.3,1)$ and $\left(C a, \omega / \dot{\gamma}_{\max }\right)=(0.3,2)$. Note that the center of mass is equivalent to the volume centroid, as opposed to the surface centroid, and the two centroids coincide for centersymmetric objects while they deviate as this symmetry is lost. In this study, the capsule symmetry is broken by the presence of the wall. The motion is a combination of oscillations in the streamwise $(x)$ direction due to the oscillating background flow and wall-normal lateral migration due to the hydrodynamic

lift. The trajectories of the two centroids, $h_{\text {lat }} / a$ and $\hat{h}_{\text {lat }}$ display significant differences: the 


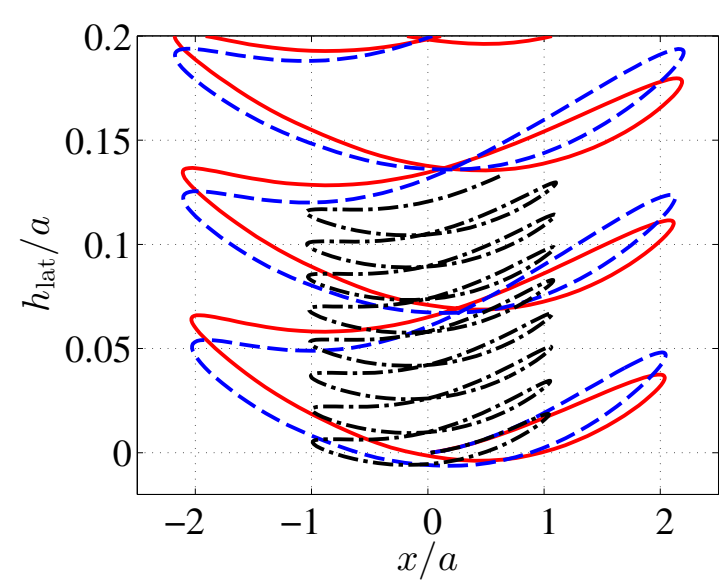

(a)

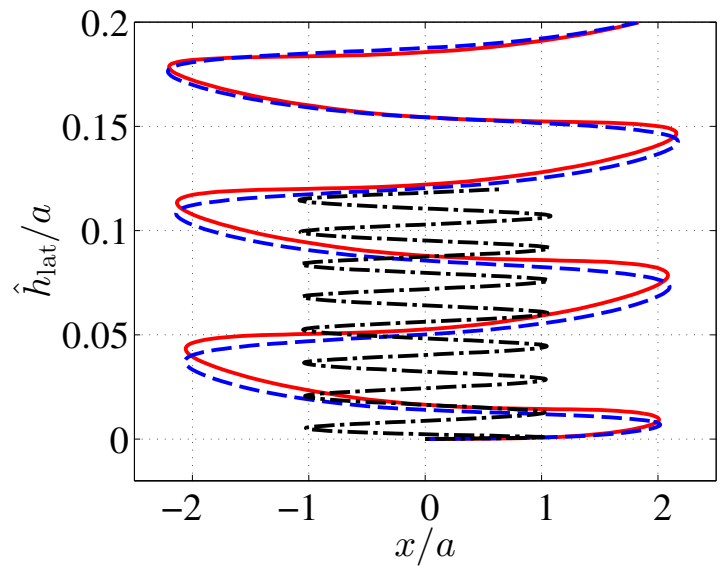

(b)

FIG. 2. (Color online) The trajectories of (a) $\left(x / a, h_{\text {lat }} / a\right)$ of the center of mass, and (b) $\left(x / a, \hat{h}_{\text {lat }} / a\right)$ of the surface centroid on the shear plane, of the capsules $\left(C a, \omega / \dot{\gamma}_{\max }\right)=(0.15,1)$ (solid), $\left(C a, \omega / \dot{\gamma}_{\max }\right)=(0.3,1)$ (dashed) and $\left(C a, \omega / \dot{\gamma}_{\max }\right)=(0.3,2)$ (dot-dashed). The capsules are released with an initial offset $h_{\text {ini }} / a=2$.

center of mass moves vertically in a reciprocal way while the surface centroid migrates away from the wall continuously.

This is also illustrated in figure 3 where we display the time migration of the two centroids, $h_{\text {lat }} / a$ and $\hat{h}_{\text {lat }} / a$. They both vary periodically with the frequency of the imposed shear, but neither resembles a regular sinusoidal as the shear: the former increases with time nonmonotonically, its migration velocity can be negative while the latter increases monotonically and hence the velocity is always positive. This difference reflects the asymmetry of the shape of the capsule. They nevertheless have approximately the same time-averaged value; after all, both centroids represent the motion of the capsule and thus coincide with each other in a statistical sense. In the following, we mostly study the time-averaged capsule migration and thus only report results for the center of mass.

We further note that a solid spherical particle will not migrate in the wall-normal direction in Stokes flow, while its ellipsoidal counterpart will exhibit an oscillatory vertical drift, with a zero mean. Nonlinearity is the cause of the particle migration, either due to inertial effects, a viscoelastic fluid or to the deformable membrane as in our case. As an example of solid particles, we note that a sphere has been shown to migrate in a constant shear flow of a viscoelastic fluid $\underline{54}$ due to the nonlinear relation between the fluid stress and the strain rate. Under oscillating shear $\underline{54}$, the spherical particle follows a wagging trajectory, very similar to that we observe here. The physical picture is also analogous to that of 


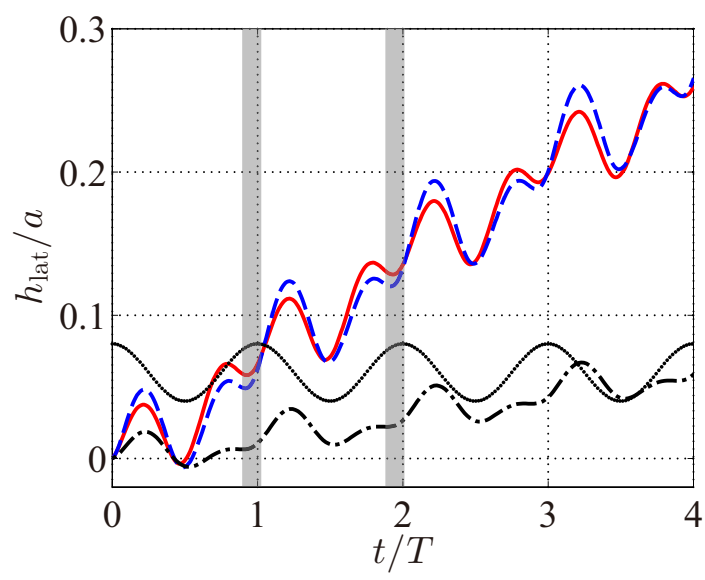

(a)

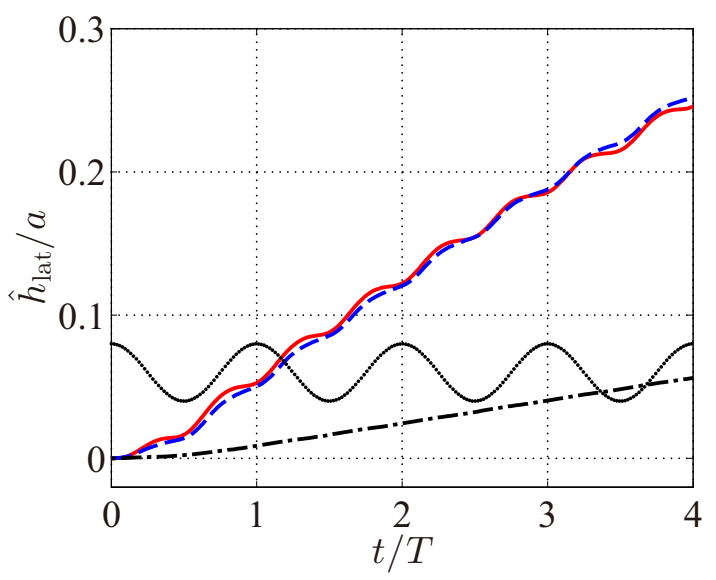

(b)

FIG. 3. (Color online) (a) Lateral migration $h_{\text {lat }} / a$, of the center of mass, and (b) $\hat{h}_{\text {lat }} / a$, of the surface centroid of the same capsules in figure 2, The dotted curve indicates the imposed periodic shear $\dot{\gamma}=\dot{\gamma}_{\max } \cos (\omega t)$, arbitrarily scaled for visualization purposes. The shaded region indicates the time when the the instantaneous lateral migration $h_{\text {lat }}$ and the shear $\dot{\gamma}$ reach the local minimum and maximum respectively.

a microswimmer exploiting nonlinearity to achieve a net locomotion despite a reciprocal motion, an interesting case in the low-Reynolds-number swimming dynamics 55 . In the Stokes regime, but in a viscoelastic or non-Newtonian fluid, a reciprocal swimming pattern can lead to a net displacement $\underline{56} \underline{-58}$, although the same strategy would not work in a Newtonian fluid 55,59 .

We further notice that for the same oscillation frequency, $\omega / \dot{\gamma}_{\max }=1$, the motion of the two capsules with $\mathrm{Ca}=0.15$ and $\mathrm{Ca}=0.3$ is similar despite the different deformability. Conversely, the trajectory of the same capsule, $\mathrm{Ca}=0.3$, varies significantly when the frequency of the imposed shear increases from $\omega / \dot{\gamma}_{\max }=1$ to $\omega / \dot{\gamma}_{\max }=2$. The streamwise domain spanned by the capsule reduces to about half when doubling $\omega / \dot{\gamma}_{\max }$, as expected since we keep the same maximum shear. The capsule lateral migration also diminishes considerably in this case, as shown in figure $3(\mathrm{~b})$.

\section{B. Velocity of the capsule}

In this section, we analyze the migration and slip velocity of the capsule. The lateral migration velocity $U_{\text {lat }}$, is computed as $U_{\text {lat }}=d h_{\mathrm{cw}} / d t$ and the slip velocity $U_{\text {slip }}=\dot{\gamma} h_{\mathrm{cw}}-U_{x}$ where $h_{\mathrm{cw}}=h_{\text {ini }}+h_{\text {lat }}$ is the distance between the capsule and the wall and $U_{x}$ the streamwise 


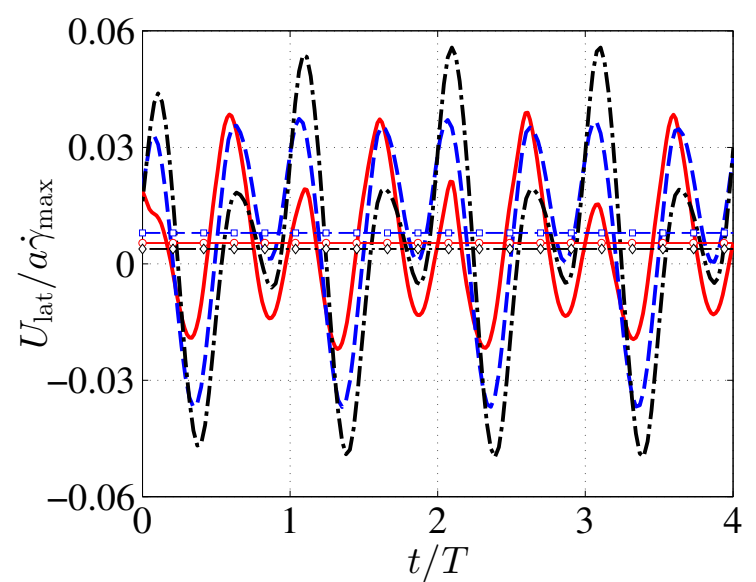

(a)

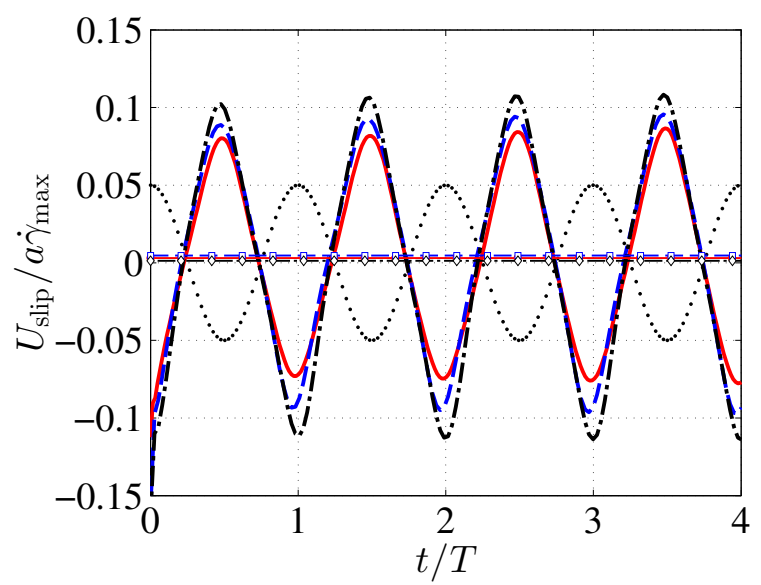

(b)

FIG. 4. (Color online) (a): Lateral migration velocity $U_{\text {lat }} / a \dot{\gamma}_{\max }$ and (b); slip velocity $U_{\text {slip }} / a \dot{\gamma}_{\max }$, as a function of the dimensional time $t / T$ for capsules with $\mathrm{Ca}=0.0375$ (solid curve), $\mathrm{Ca}=0.15$ (dashed) and $\mathrm{Ca}=0.6$ (dot-dashed), released with an initial offset $h_{\text {ini }} / a=2$; the oscillation frequency of the imposed shear $\omega / \dot{\gamma}_{\max }=5 / 3$. The time-averaged migration and slip velocities are denoted by horizontal lines with circles $(\mathrm{Ca}=0.0375)$, squares $(\mathrm{Ca}=0.15)$ and diamonds $(\mathrm{Ca}=0.6)$. The dotted curve indicates the applied oscillating shear $\dot{\gamma}_{\max } \cos (\omega t)$, arbitrarily scaled for visualization purposes.

velocity of the center of the capsule. The temporal evolution of $U_{\text {lat }} / a \dot{\gamma}_{\max }$ and $U_{\text {slip }} / a \dot{\gamma}_{\max }$ are depicted in figure 4(a) and figure 4(b) for three capsules with the same initial offset $h_{\text {ini }} / a=2$ and frequency of the imposed shear, $\omega / \dot{\gamma}_{\max }=5 / 3$. After an initial transient, the two velocities vary periodically in time. The transient is also observed in the case of steady shear $\stackrel{41}{ }$, before the capsule reaches a quasi-steady state; only then its deformation and velocity do not vary with the instantaneous capsule-wall distance $h_{\mathrm{cw}}$. In the case of periodic shear investigated here, the capsule motion reaches a quasi-periodic regime. Unless otherwise specified, we only examine its dynamics at this stage, when its net migration $h_{\text {lat }}$ is negligible compared to the initial offset $h_{\mathrm{ini}}$ and $h_{\mathrm{ini}} \approx h_{\mathrm{cw}}$. In the following, $h_{\mathrm{ini}}$ will therefore be used to denote the capsule-wall distance.

As clear from figure 4(b), the slip velocity and the background shear flow have different signs. This indicates that the capsule moves faster than the local background flow, an effect more pronounced when $t / T$ approximately assumes integer values and the capsule returns about the initial location $(x=0)$. This is different to what is observed in a steady shear flow where the capsule always lags behind the surrounding fluid $\underline{41} \underline{42}$. In fact, as 


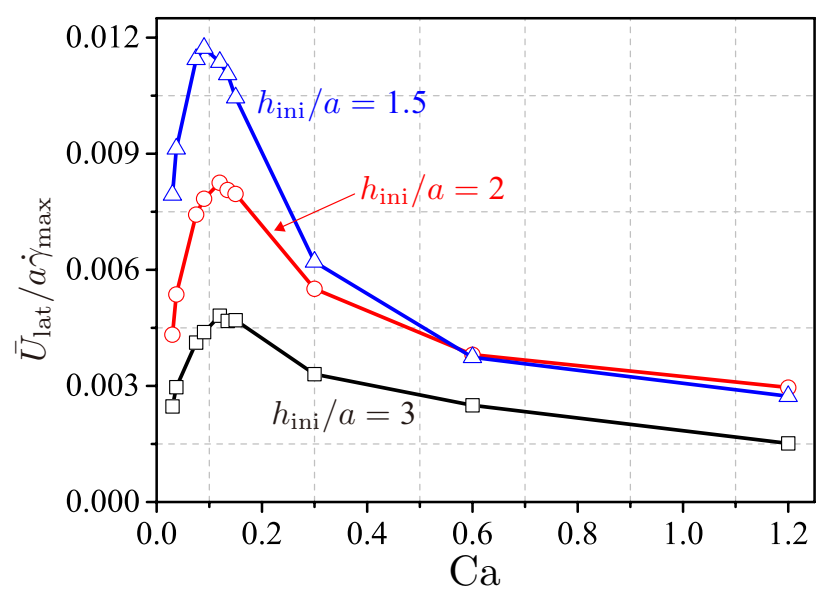

FIG. 5. (Color online) Lateral migration velocity $U_{\text {lat }} / a \dot{\gamma}_{\max }$ as a function of the dimensional time $t / T$ and the capillary number Ca, at fixed oscillation frequency $\omega / \dot{\gamma}_{\max }=5 / 3$. Data are reported for three initial offsets, $h_{\text {ini }} / a=1.5, h_{\text {ini }} / a=2$ and $h_{\text {ini }} / a=3$ indicated by the triangles, circles and squares respectively.

$t / T \approx 1,2,3 \ldots$ (shaded regions in figure $3(\mathrm{~b})$, the capsule-wall distance $h_{\mathrm{cw}}=h_{\mathrm{ini}}+h_{\text {lat }}$ is at a local minimum and the background shear $\dot{\gamma}$ reaches its maximum value. At this time, the background velocity $\dot{\gamma} h_{\mathrm{cw}}$, the product of the two, decreases because the phase difference is around $\pi$. This phase difference does not exist in steady shear when there are no delays and the local background flow depends only on the capsule-wall distance $h_{\mathrm{cw}}$.

The time-averaged value of the migration velocity $\bar{U}_{\text {lat }} / a \dot{\gamma}_{\max }$ and of the slip velocity $U_{\text {slip }} / a \dot{\gamma}_{\text {max }}$ of the three capsules are indicated in figure 4 by horizontal lines. For all cases, the mean migration velocity is positive: the capsule undergoes a net migration away from the wall. However, the mean slip velocity is almost zero, namely, the capsule has no net motion in the streamwise direction. The capsule with the intermediate capillary number $\mathrm{Ca}=0.15$ has a higher migration velocity than its floppy $(\mathrm{Ca}=0.0375)$ and stiff $(\mathrm{Ca}=0.6)$ counterparts. To further examine this dependence, the migration velocity $\bar{U}_{\text {lat }} / a \dot{\gamma}_{\max }$ is therefore displayed versus the capillary number $\mathrm{Ca}$ in figure 5 for three different initial offsets. The mean velocity varies non-monotonically with $\mathrm{Ca}$, the optimal capillary number $\mathrm{Ca}_{\text {opt }}$ being around 0.1. This non-monotonic dependence of the migration velocity is in contrast to what is observed in steady shear, where the lift velocity increases monotonically with $\mathrm{Ca}^{37,41,60}$.

In steady flows, the particle lateral migration velocity is a linear function of the normal stress differences $N_{1}-N_{2}$ as shown among others in Ref. 60 and 37. It is thus natural to investigate whether this relation holds in an oscillating shear as well. The normal stresses 
$N_{1}$ and $N_{2}$ are defined as,

$$
\begin{aligned}
& N_{1}=\sigma_{x x}-\sigma_{y y}, \\
& N_{2}=\sigma_{y y}-\sigma_{z z},
\end{aligned}
$$

where $\boldsymbol{\sigma}$ denotes the extra stress due to the presence of the capsule in the volume $V$ :

$$
\sigma_{i j}=-\frac{1}{V} \int_{S} \boldsymbol{\rho}_{i}^{\mathrm{e}} x_{j} d S .
$$

We consider the nondimensional stress difference $\left(N_{1}-N_{2}\right) / \mu \phi_{\mathrm{v}} \dot{\gamma}_{\max }$, with $\phi_{\mathrm{v}}=1 / V$ the volume fraction; in the dilute limit, the normal stress difference $\Delta N=\left(N_{1}-N_{2}\right) / \mu \phi_{\mathrm{v}} \dot{\gamma}_{\max }$ represents the capsule-induced stress normalized by the viscous stress.

The temporal evolution of the migration velocity $U_{\text {lat }} / a \dot{\gamma}_{\max }$ and the normal stress difference $\Delta N$ are depicted in the top panel of figure 6 for capsules with $\mathrm{Ca}=0.075, \mathrm{Ca}=0.12$ and $\mathrm{Ca}=0.6$. After the transient period, both quantities vary periodically and reach their local maxima with small phase delays, which indicates a considerable correlation between these two quantities. The period is around $T / 2$, indicating that $U_{\text {lat }}$ and $\Delta N$ change twice as faster as the oscillating shear.

We next examine the local maxima of $U_{\text {lat }} / a \dot{\gamma}_{\max }$ (marked by circles in the figure) and of $\Delta N$ (indicated by squares), always neglecting the peaks in the transient period. For the stiff capsule $\mathrm{Ca}=0.075$ (cf. figure 6(a), we identify five instants when $U_{\text {lat }} / a \dot{\gamma}_{\max }$ and $\Delta N$ both attain local maxima. At times $t_{1}, t_{3}$ and $t_{5}$, the peaks of the two quantities have a similar magnitude with respect to their own scales, while at the time $t_{2}$ and $t_{4}$, their relative magnitude is significantly different. In other words, the ratio between the magnitude of the two local maxima varies with time, something which becomes more evident for the floppy capsule $\mathrm{Ca}=0.6$. In contrast, the two quantities $U_{\text {lat }} / a \dot{\gamma}_{\max }$ and $\Delta N$ have roughly the same magnitude every time they reach a local maxima for the capsule with $\mathrm{Ca}=0.12$, the one displaying the largest lateral migration velocity (see figure 6(b)]: the ratio of the peak values remains almost constant in time. This implies that the correlation between the two quantities is stronger when $\mathrm{Ca} \approx 0.1$ and decreases for larger and smaller values of the capillary number $(\mathrm{Ca}=0.075$ and 0.6 in the figure). This high correlation suggests that the normal stress difference contributes to a more pronounced lateral migration.

To gain further insight, we show the time-averaged migration velocity $\bar{U}_{\text {lat }} / a \dot{\gamma}_{\text {max }}$ together with the time-averaged normal stress difference $\Delta^{-} N$ versus the capillary number Ca in figure 7, the data reported pertain to three different values of the initial distance from the wall. Interestingly, these two quantities follow closely the same trend: a sharp increase with 


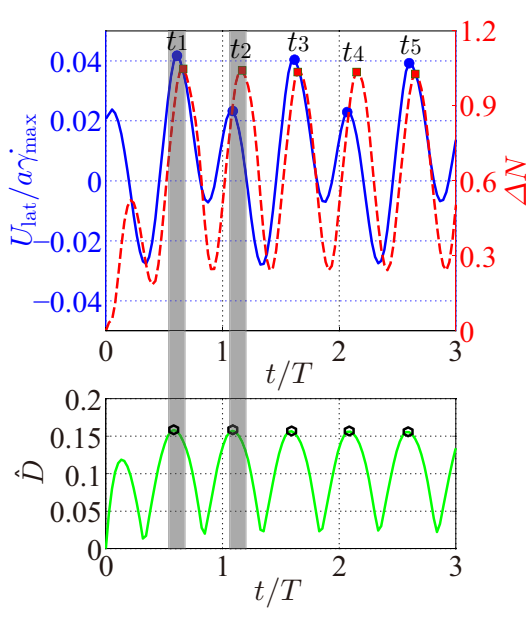

(a)

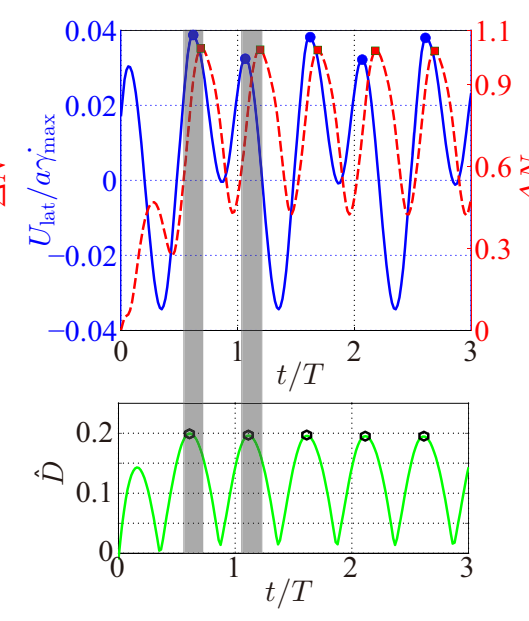

(b)

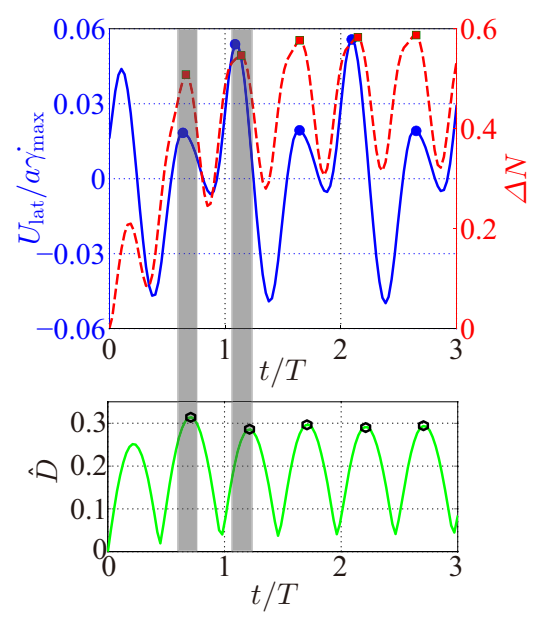

(c)

FIG. 6. (Color online) Top panel: lateral migration velocity, $U_{\text {lat }} / a \dot{\gamma}_{\max }$, (solid lines) and normal stress difference $\Delta N$ (dashed lines) versus the nondimensional time $t / T$, for three capillary numbers $\mathrm{Ca}=0.075(\mathrm{a}) 0.12$ (b) and $0.6(\mathrm{c})$. Bottom panel: deformation index $\hat{D}$ versus $t / T$ for the same capsule. The initial offset is $h_{\mathrm{ini}} / a=2$ and the frequency of the imposed shear $\omega / \dot{\gamma}_{\max }=5 / 3$. The local maxima of the velocity, stress and deformation are marked by the circles, squares and hexagons respectively; the shaded region indicates the period when they co-appear.

Ca for stiff capsules, a maximum at $\mathrm{Ca} \approx 0.1$ and then slower decrease for softer and softer membranes.

The parameter study is continued by examining the dependence of the lateral migration velocity on the frequency of oscillation of the imposed shear, $\omega / \dot{\gamma}_{\max }$. Figure 8 displays the time-averaged migration velocity, $\bar{U}_{\text {lat }} / a \dot{\gamma}_{\text {max }}$, versus $\omega / \dot{\gamma}_{\text {max }}$ for the capsule with Ca $=0.3$ and the three offsets $h_{\text {ini }} / a=1.5,2$ and 3. As $\omega / \dot{\gamma}_{\max }<2, \bar{U}_{\text {lat }} / a \dot{\gamma}_{\text {max }}$ decreases sharply with $\omega / \dot{\gamma}_{\max }$, almost linearly; for $\omega / \dot{\gamma}_{\max }>2$, the migration velocity decreases more slowly, approaching asymptotically zero for large $\omega / \dot{\gamma}_{\max }$. The cases with $\omega / \dot{\gamma}_{\max }=0$ correspond to a capsule in steady shear flow. Conversely, as $\omega / \dot{\gamma}_{\max } \rightarrow \infty$, the flow oscillations are much faster than the relaxation time of the capsule and the capsule therefore does not have sufficient time to adjust to the flow before it changes direction: the deformation and the consequent lateral migration are hence negligible.

The variations of the time-averaged migration velocity $\bar{U}_{\text {lat }} / a \dot{\gamma}_{\text {max }}$ with the oscillation frequency are displayed in figure 9 versus the capillary number Ca for capsules with an initial offset $h_{\text {ini }} / a=2$. The non-monotonic variation of $\bar{U}_{\text {lat }} / a \dot{\gamma}_{\text {max }}$ with Ca holds for all cases, but the capillary number $\mathrm{Ca}_{\mathrm{opt}}$ of maximum lateral velocity changes with the frequency 


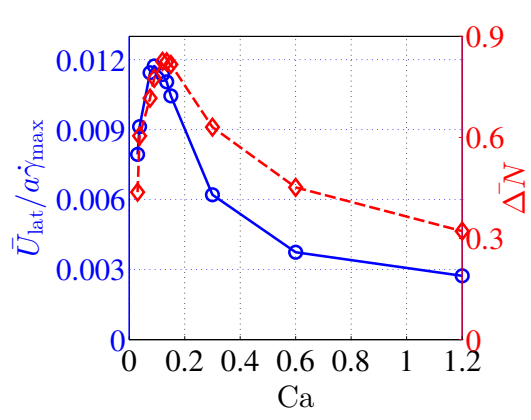

(a)

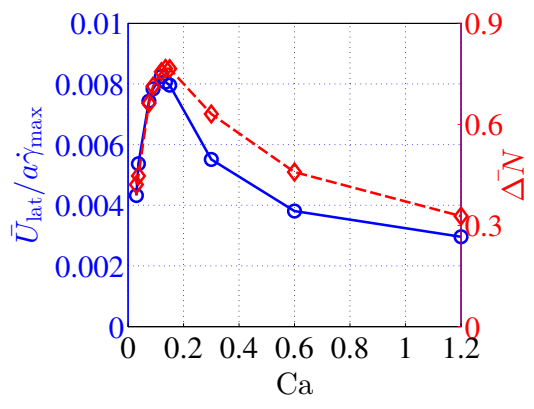

(b)

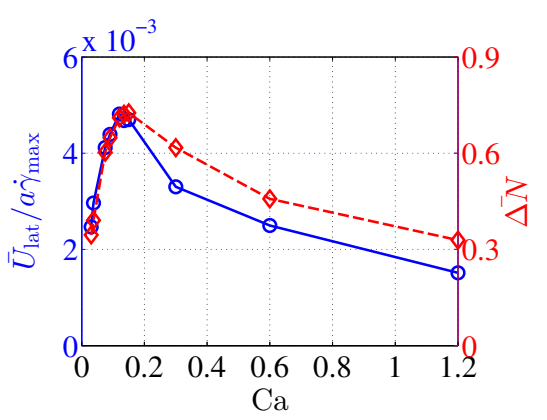

(c)

FIG. 7. (Color online) Time-averaged value of the lateral migration velocity $\bar{U}_{\text {lat }} / a \dot{\gamma}_{\max }$ and of the normal stress differences $\Delta \overline{\Delta N}$, versus the capillary number Ca. $\bar{U}_{\text {lat }} / a \dot{\gamma}_{\max }$ and $\overline{\Delta N}$ are indicated by the solid (resp. dashed) curve with circles (resp. diamonds), measured by the left (resp. right) scale. The frequency of the shear is $\omega / \dot{\gamma}_{\max }=5 / 3$ and the results are shown for (a) $h_{\text {ini }} / a=1.5$, (b) $h_{\text {ini }} / a=2$ and (c) $h_{\text {ini }} / a=3$.

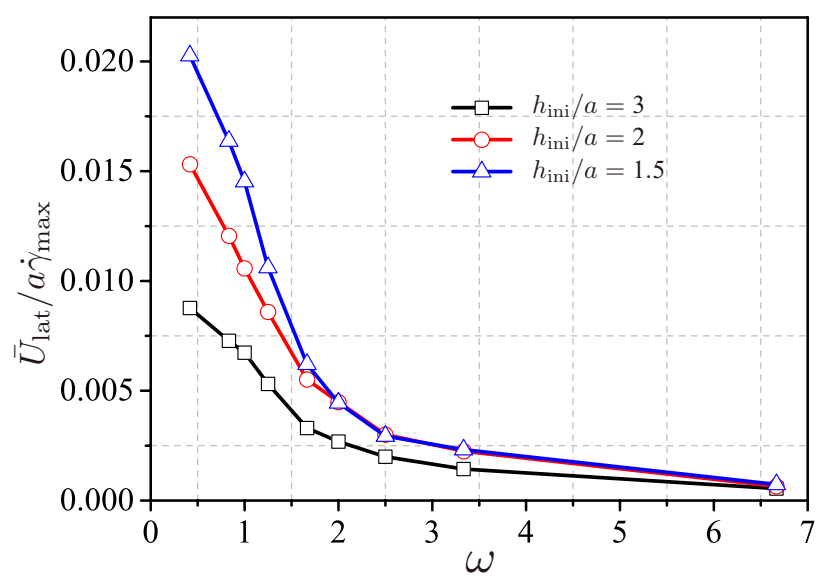

FIG. 8. (Color online) Time-averaged migration velocity $\bar{U}_{\text {lat }} / a \dot{\gamma}_{\max }$, as a function of the frequency of the oscillating shear $\omega / \dot{\gamma}_{\max }$. The capillary number $\mathrm{Ca}$ of the capsule is 0.3 and three initial positions of the capsules are chosen, $h_{\text {ini }} / a=3$ denoted by squares, $h_{\text {ini }} / a=2$ by circles and $h_{\text {ini }} / a=1.5$ by triangles.

$\omega / \dot{\gamma}_{\max }$. The inset of the same figure shows that $\mathrm{Ca}_{\text {opt }}$ varies almost linearly with the inverse of the shear frequency $\dot{\gamma}_{\max } / \omega$. In fact, as the migration velocity, normal stress difference and capsule deformation vary at a frequency of roughly $2 \omega / \dot{\gamma}_{\max }$ (see figure. 6), $2 \omega / \dot{\gamma}_{\max }$ can be considered as the effective frequency of the flow oscillation determining the capsule behavior. Accordingly, the ratio of the relaxation time of the capsule over the effective time scale of the flow is $2 \mathrm{Ca} \omega / \dot{\gamma}_{\max }$. 
The inset indeed shows that $2 \mathrm{Ca}_{\mathrm{opt}} \omega / \dot{\gamma}_{\max } \approx 0.5$. In another words, lateral migration becomes strongest as the effective time scale of the flow is of the order of the relaxation time of the elastic membrane.

It is worth noting that this scaling has similarities with that observed in many other situations where the flow dynamics is significantly influenced by the nonlinearity solely arising from the elasticity of the fluid or structure. A rotating helical slender body, a typical model micro-swimmer propelling like a cork-screw, attains the most efficient propulsion when the relaxation time of the polymeric fluid it is immersed in is of the order of the typical flow time ${ }^{61,62}$. Similarly, an elastic filament $\underline{63}, \underline{64}$ or flapper ${ }^{65}$ actuated in a viscous fluid for propulsion or pumping reaches maximum efficiency as the so-called sperm number ${ }^{66}$, the ratio of the time scale of the elastic structure over that of the flow, is of order one. We further note that the relation between the optimal capillary number and the frequency of the applied shear could be potentially used to aid the design of flow-assisted devices to sort deformable cells $\underline{67}-\underline{69}$. Specific cells may be extracted from a dilute suspension in an oscillating Couette device if the frequency of the applied shear is tuned to match the capillary number of the targeted cells; these cells would in fact migrate with highest velocity. The frequency of the applied shear would have to depend on the deformability of the targeted cells, therefore the same device can be used to sort cells with different deformabilities by varying the frequency of operation.

Furthermore, the relation $2 \mathrm{Ca}_{\text {opt }} \omega / \dot{\gamma}_{\max } \approx 0.5$ can also be implied theoretically in the limit of high-frequency oscillation, i.e. $\omega / \dot{\gamma}_{\max } \gg 1$, when the capsule undergoes small deformation. We can assume the capsule reaches the peak deformation and migration velocity at the same capillary number $\mathrm{Ca}_{\mathrm{opt}}$. In an unbounded oscillating shear, the leading order of peak deformation is $\frac{43}{D_{\text {peak }}}=\frac{1}{2 \omega / \dot{\gamma}_{\max }}$ and that of the equilibrium value $D$ in the steady case is $\frac{3.70}{-70}=\frac{25}{12} \mathrm{Ca}$. Hence the capsule reaches the peak deformation and migration as $2 \mathrm{Ca}_{\mathrm{opt}} \omega / \dot{\gamma}_{\max }=\frac{12}{25} \approx 0.5$.

Finally, we report in figure 10 the time-averaged migration velocity $\bar{U}_{\text {lat }} / a \dot{\gamma}_{\text {max }}$ and the normal stress difference versus the capillary number $\mathrm{Ca}$ for two different oscillation frequencies, $\omega / \dot{\gamma}_{\max }=0.5$ and $\omega / \dot{\gamma}_{\max }=3$. As shown in figure 7 , we can clearly identify a positive correlation between these two quantities. 


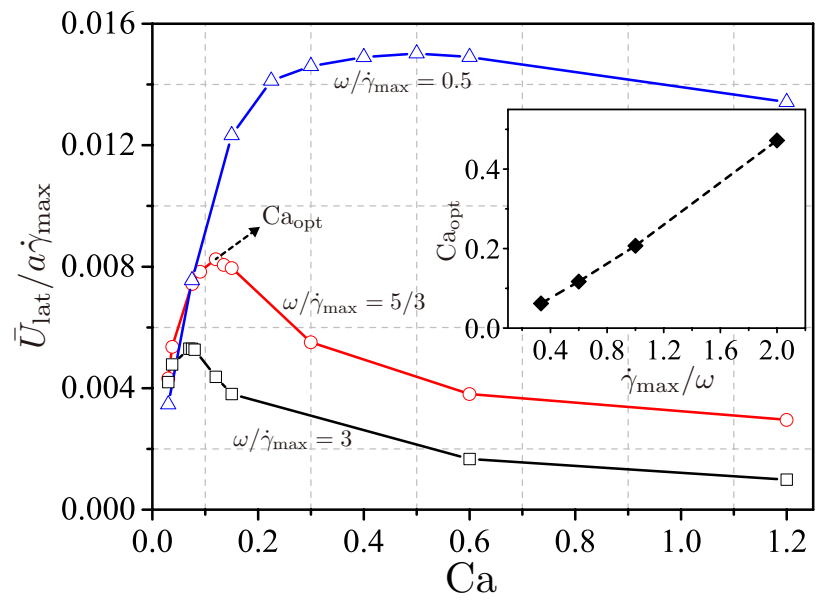

FIG. 9. (Color online) The time-averaged lateral migration velocity $\bar{U}_{\text {lat }} / a \dot{\gamma}_{\text {max }}$ versus the capillary number Ca, for three shear frequencies, $\omega / \dot{\gamma}_{\max }=0.5$ (triangles), $\omega / \dot{\gamma}_{\max }=5 / 3$ (circles) and

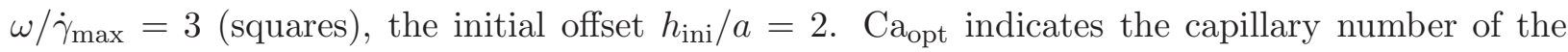
capsule with the maximum migration velocity and the inset shows its dependence on $\dot{\gamma}_{\max } / \omega$.

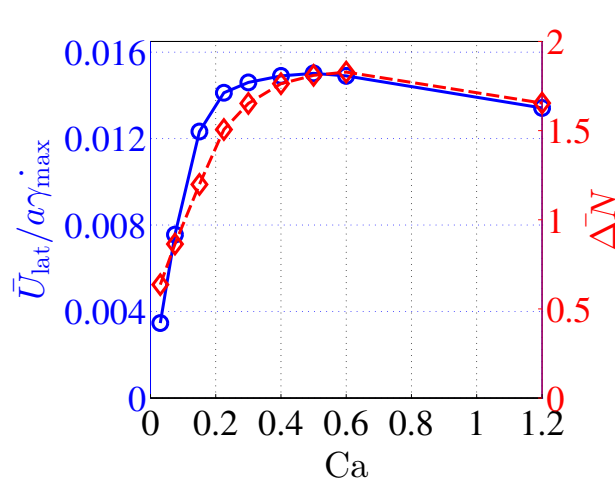

(a)

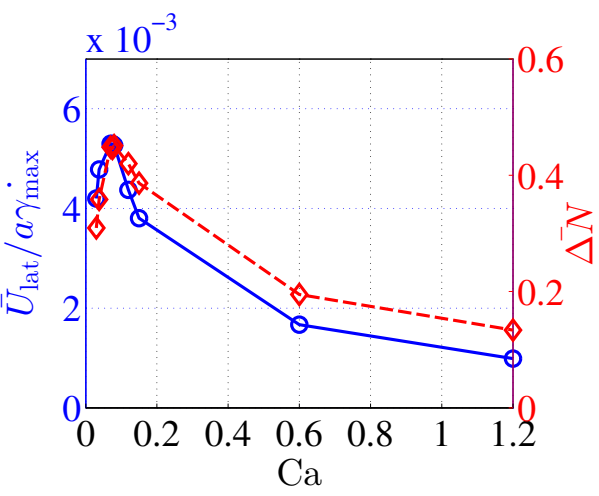

(b)

FIG. 10. (Color online) The time-averaged value of the migration velocity $\bar{U}_{\text {lat }} / a \dot{\gamma}_{\text {max }}$ and that of the normal stress differences $\Delta^{-} N$ versus the capillary number Ca. The initial offset is $h_{\text {ini }} / a=2$ : (a) $\omega / \dot{\gamma}_{\max }=0.5$ and (b): $\omega / \dot{\gamma}_{\max }=3$.

\section{Deformation of the capsule}

A capsule typically evolves into a prolate when subject to an unbounded shear flow. Hence, its deformation is usually quantified by the so-called Taylor parameter $D=\left(L_{\max }-\right.$ $\left.L_{\min }\right) /\left(L_{\max }+L_{\min }\right)^{\underline{44}}$, where $L_{\max }$ and $L_{\min }$ are the length of the major and minor axis of the elliptical profile in the shear plane. In our case, the profile of the capsule on the shear plane is not a ellipse, since the symmetry is broken by the presence of the wall as also observed by 


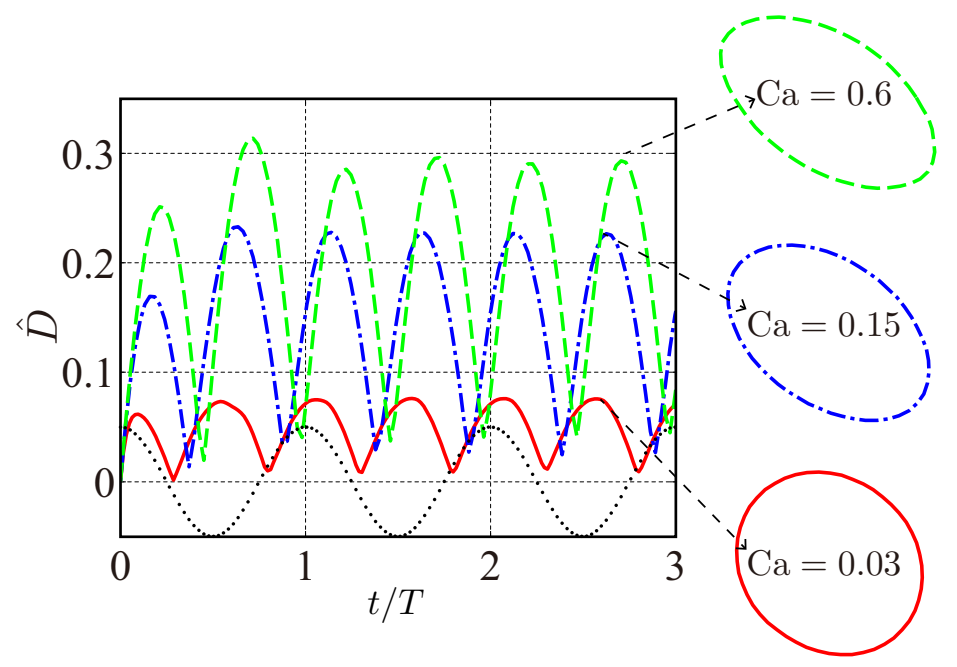

FIG. 11. (Color online) Temporal evolution of the deformation index $\hat{D}$ for capsules with initial offset $h_{\mathrm{ini}} / a=2$ and frequency of the shear $\omega / \dot{\gamma}_{\max }=5 / 3$. Results for $\mathrm{Ca}=0.03, \mathrm{Ca}=0.15$ and $\mathrm{Ca}=0.6$ are indicated by the solid, dot-dashed and dashed lines respectively. The dotted curve denotes the oscillating background shear $\dot{\gamma}_{\max } \cos (\omega t)$, arbitrarily scaled for a better visualization. The profiles of the capsules reaching the peak deformation are displayed on the shear plane.

Nix et al $\underline{\underline{42}}$. We thus introduce the deformation index, $\hat{D}=\left(L_{\max }^{\prime}-L_{\min }^{\prime}\right) /\left(L_{\max }^{\prime}+L_{\min }^{\prime}\right)$, where $L_{\max }^{\prime}$ is the maximum distance measured from the capsule surface to the its center of mass and $L_{\min }^{\prime}$ is the minimum distance. The deformation index $\hat{D}$ quantifies how far away the capsule is from its stress-free shape, a sphere in the current case.

The time evolution of the deformation index $\hat{D}$ is illustrated in figure 11 for three values of the capillary number, $\mathrm{Ca}=0.03,0.15$ and 0.6 , same initial offset, $h_{\text {ini }} / a=2$, and shear frequency $\omega / \dot{\gamma}_{\max }=5 / 3$. The index $\hat{D}$ displays wake-like variations in time. An initial overshoot is observed for the soft capsules, $\mathrm{Ca}=0.3$ and 0.6 , similar to that observed in the wall-bounded steady shear flow $\underline{\underline{41}}$. After one or two periods, the amplitude of the oscillations becomes constant. The initial transient is more evident for the relatively soft capsules, $\mathrm{Ca}=0.15$ and 0.6 ; indeed, a higher capillary number implies a longer relaxation time, hence the capsule needs more time to adapt to the unsteady flow. We also note in the figure that the frequency of the periodically varying deformation index $\hat{D}$ is twice that of the background shear. The stiffest capsule, $\mathrm{Ca}=0.03$, displays the maximum deformation roughly after the local extrema of the shear and the minimum deformation as the flow reverses its direction. The stiff capsule therefore feels and responds to the variations of the flow fast enough to change its shape accordingly; following so closely the flow, its maximum 


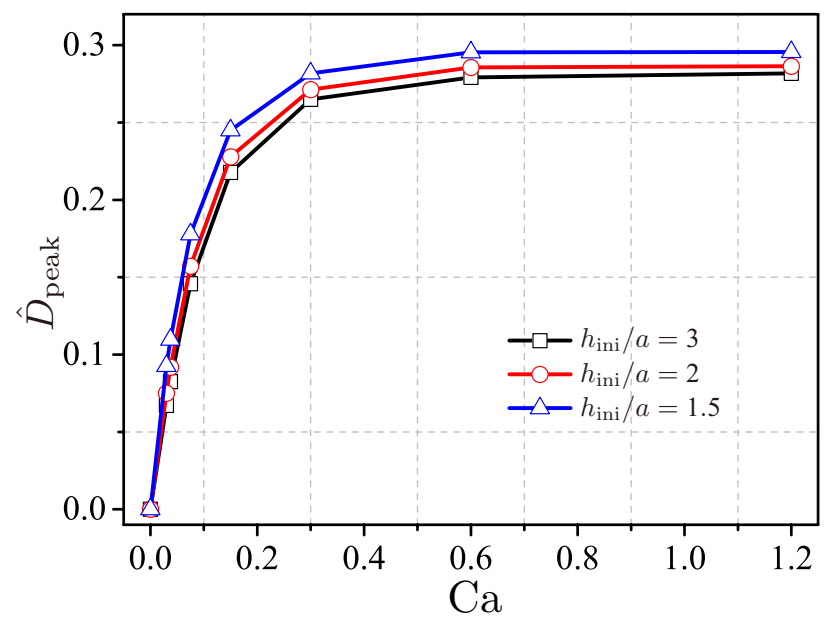

FIG. 12. (Color online) The temporal peak of deformation $\hat{D}_{\text {peak }}$ versus the capillary number Ca, for an initial offset $h_{\text {ini }} / a=1.5,2$ and 3. The shear frequency is $\omega / \dot{\gamma}_{\max }=5 / 3$.

deformation appears very shortly after the instant of maximum shear. The opposite applies to softer capsules.

The temporal evolution of the capsule deformation was also reported in the bottom panel of figure 6. The shaded regions clearly indicate that the local maxima of the migration velocity, normal stress difference and deformation index appear roughly at a same moment; moreover, the three quantities vary with twice the frequency of the background shear. Such a coincidence is not surprising; in fact, for a droplet and a capsule in steady shear, theoretical $^{71}$ and numerical $\underline{41}$ works have identified the relation between its migration velocity and deformation as $U_{\text {lat }} \sim \hat{D}\left(a / h_{\text {ini }}\right)^{2}$. Our analysis further confirmed that even in the presence of flow oscillation, the deformation and migration velocity, which are time-dependent in this case, are correlated.

To better understand the capsule deformation, we examine the peak deformation $\hat{D}_{\text {peak }}$, the maximum value of $\hat{D}$ after the initial transient overshoot. Figure 12 depicts $\hat{D}_{\text {peak }}$ as a function of the capillary number Ca for the same three initial heights considered above. $\hat{D}_{\text {peak }}$ increases almost linearly with the capillary number $\mathrm{Ca}$ for $\mathrm{Ca}<0.1$. This resembles the linear relation between the Taylor parameter $D$ and the capsule capillary number in an unbounded steady shear flow. Further increasing the capillary number, $\hat{D}_{\text {peak }}$ increases more slowly and reaches an asymptotic value close to 0.3 as $\mathrm{Ca}>0.6$. This is in contrast to what observed in an unbounded steady shear where the deformation $D$ always increases with $\mathrm{Ca}$ although slowly at large Ca.

In oscillating flows, the deformation not only depends on Ca but also on the frequency 


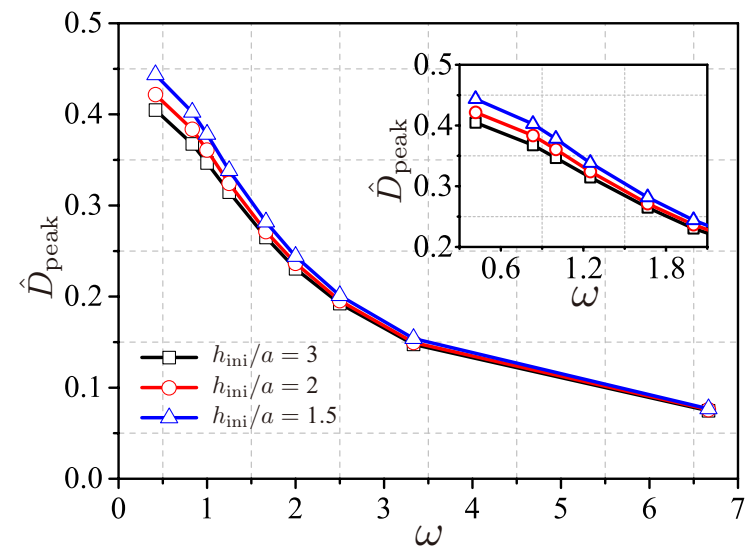

(a)

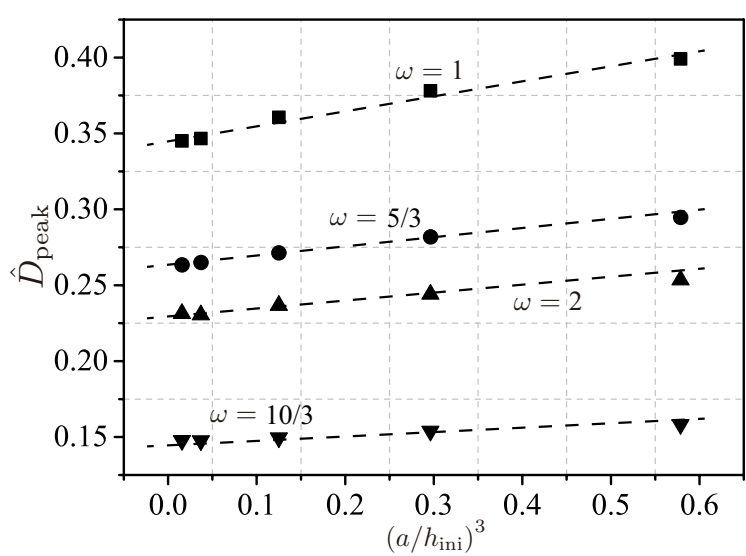

(b)

FIG. 13. (Color online) (a) the peak deformation $\hat{D}_{\text {peak }}$ as a function of the shear frequency $\omega / \dot{\gamma}_{\text {max }}$, for $h_{\text {ini }} / a=1.5,2$ and 3 and capillary number $\mathrm{Ca}=0.3$. (b). $\hat{D}_{\text {peak }}$ versus $\left(a / h_{\text {ini }}\right)^{3}$ for $\omega / \dot{\gamma}_{\max }=1,5 / 3,2$ and $10 / 3$.

of the oscillations, $\omega / \dot{\gamma}_{\text {max }}$. The time needed to reach the maximum deformation increases with $\mathrm{Ca}$; for large values of $\mathrm{Ca}$, the capsule fails to reach the maximum possible deformation that would occur in a steady shear before the flow changes direction if the oscillations occur fast enough. Considering $T / 2$, the time during which the shear has constant direction, the deformation of the capsules is hence limited by $T$ instead of by its deformability for large values of the shear oscillation frequency. The relation between the deformation and $\omega / \dot{\gamma}_{\max }$ can also explain the non-monotonic dependence of the migration velocity with Ca. The lateral migration velocity, $U_{\text {lat }}$, of a capsule with $\lambda=1$ in wall-bounded shear is related to $S_{\text {mem }}^{y y}$, the $y y$ component of the stresslet $\mathbf{S}_{\text {mem }}$ induced by the elastic force on the membrane by $\frac{41,42}{2}$

$$
\frac{U_{\text {lat }}}{a \dot{\gamma}}=-\frac{9}{64 \pi}\left(\frac{a}{h_{\mathrm{cw}}}\right)^{2} \frac{S_{\mathrm{mem}}^{y y}}{G_{\mathrm{s}} a^{2}} \frac{1}{\mathrm{Ca}},
$$

where $S_{\text {mem }}^{y y}=\int_{S}-\rho_{y}^{\mathrm{e}}(\mathbf{x})\left(\mathbf{x}_{y}-\mathbf{x}_{y}^{\mathrm{cen}}\right) d S(\mathbf{x})$ and $\frac{S_{\mathrm{mem}}^{y y}}{G_{s} a^{2}}$ depends only on the capsule deformation. This increases faster at low $\mathrm{Ca}$ and more slowly as $\mathrm{Ca}>0.2$ (see figure. 12) and so does $\frac{S_{\text {mem }}^{y y}}{G_{\mathrm{s}} a^{2}} ; \frac{1}{\mathrm{Ca}}$, conversely, decreases so that the product of the two decreases when Ca is above a certain value. In another words, the lateral migration of high-Ca capsules is hampered by the limiting deformation they attain.

We further examine the dependence of $\hat{D}_{\text {peak }}$ on the frequency of the shear $\omega / \dot{\gamma}_{\max }$ and on the initial height $h_{\text {ini }} / a$ in figure 13, where the membrane deformability is kept at Ca $=$ 0.3. The maximum deformation $\hat{D}_{\text {peak }}$ decreases monotonically with $\omega / \dot{\gamma}_{\max }$ as shown in figure 13(a). $\hat{D}_{\text {peak }}$ increases as $h_{\text {ini }} / a$ decreases, i.e. larger confinement effects, and this is 


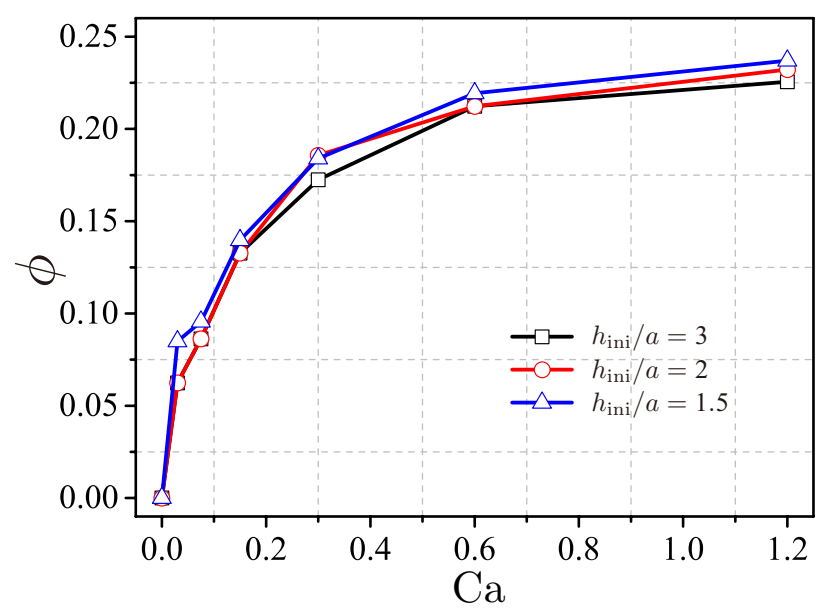

FIG. 14. (Color online) Phase delay $\phi$ of the capsule deformation with respect to the applied shear versus the capillary number Ca; the frequency of shear is $\omega / \dot{\gamma}_{\max }=5 / 3$. Results are shown for an initial offset $h_{\text {ini }} / a=1.5$ (triangles), 2 (circles) and 3 (squares).

more evident for smaller values of $\omega / \dot{\gamma}_{\text {max }}$; for large $\omega / \dot{\gamma}_{\max }$, the deformation barely varies with the initial height for the same mechanism explained above. We show in figure 13(b) that the maximum deformation $\hat{D}_{\text {peak }}$ is proportional to $\left(a / h_{\text {ini }}\right)^{3}$. Note that in a wall-bounded steady shear flow, the same power law has been identified for the deformation of a droplet by Shapira and Haber ${ }^{72}$ theoretically and for a capsule by Singh et al. $\underline{41}$ using numerical simulations.

To quantify the delay between the capsule deformation and the shear oscillations, we define the time difference $\left(t_{\operatorname{maxD}}-t_{\max }\right)$ scaled by the characteristic flow time as the phase lag $\phi=\left(t_{\operatorname{maxD}}-t_{\operatorname{maxS}}\right) / T$. The phase delay $\phi$ is negligible for the stiff capsule,whereas it is $\phi \approx 1 / 4$ for the floppy capsule with $\mathrm{Ca}=0.6$; the latter deforms most when the flow changes direction (see the time histories in figure 11). Note again that, regardless of the difference in the phase delay between capsules of different capillary numbers, the deformation oscillates at a frequency around $2 \omega / \dot{\gamma}_{\text {max }}$.

Finally, we examine in figure 14 the dependence of the phase delay $\phi$ on the capillary number Ca. The phase delay $\phi$ does not vary significantly with the initial offset $h_{\text {ini }} / a$ of the capsules. $\phi$ increases monotonically with $\mathrm{Ca}$, an effect more pronounced when $\mathrm{Ca}$ is below 0.2 . The delay $\phi$ reaches an asymptotic value of approximately 0.25 as Ca continues to increase. Note that the phase delay $\phi$ assumes a value between 0 (for purely viscous fluid) and 0.5 (for a linearly elastic solid). The asymptotic value of $\approx 0.25$ clearly indicates that viscoelastic effect is an important feature in the flow of deformable cells. 


\section{CONCLUSIONS}

Using an accelerated boundary integral method for the flow coupled with a global spectral method for the membrane, we study the near-wall dynamics of an initially spherical capsule with neo-Hookean membrane in an oscillating shear flow. We focus on the lateral migration and deformation of the capsule, and their dependence on the capillary number, the frequency of the background shear and the initial wall-capsule distance.

The shape of capsule is asymmetric due to the presence of one wall and hence its center of mass and surface centroid are not equivalent, as discussed in Ref. 42. The time history of the lateral migration of the two centroids are clearly different, their time-averaged migrations being however similar. It is thus important to specify the definition of the centroids of asymmetric deformable particles when quantifying their migrations.

The capsule reaches a quasi-periodic steady state after an initial transient period. During this state, the capsule follows a wiggling trajectory, moving away from and towards the wall periodically, with a net lateral migration away from the wall. For the capsule-wall distances and shear frequencies investigated here, the mean migration velocity varies nonmonotonically with the capillary number $\mathrm{Ca}$, reaching the maximum migration at an optimal capillary number denoted $\mathrm{Ca}_{\mathrm{opt}}$.

The optimal capillary number $\mathrm{Ca}_{\mathrm{opt}}$ is sensitive to the frequency of the shear, and is shown to scale linearly with the inverse of $\omega / \dot{\gamma}_{\max }$. Interestingly, it does not vary significantly with the capsule-wall distance. The maximum migration is observed when the effective oscillation period is of the order of the relaxation time of the elastic membrane.

The capsule lateral migration velocity decreases monotonically with the frequency of the imposed shear; at relatively high oscillation rates, the capsule fails to adapt to the flow before it changes direction so that its lateral migration and surface deformation become negligible.

The relation between the lateral migration velocity and the normal stress difference induced in the flow is explored. The maxima of these two quantities are closely correlated, with a weak time delay function of the capillary number. The relative magnitude of the correlations can vary significantly. $\mathrm{As} \mathrm{Ca} \approx \mathrm{Ca}_{\text {opt }}$, when the capsule has the highest migration velocity, the correlation is highest. The dependency of these two quantities on the capillary number $\mathrm{Ca}$ is investigated for different capsule-wall distances and oscillation frequencies of the imposed shear; in all cases, the two curves agree well with each and the peak values occur as $\mathrm{Ca} \approx \mathrm{Ca}_{\mathrm{opt}}$. This confirms the correlation previously observed in steady wall-bounded 
shear flows $\frac{37}{3}$. It is worth pointing that such a relationship has also been discovered for a vesicle in two-dimensional unbounded Couette flow $\underline{73}$; the vesicle migrates towards the center at a velocity linearly scaling with the normal stress difference.

The deformation of the capsule exhibits a periodic variation approximately in phase with that of the migration velocity and of the normal stress difference, also at twice the frequency of the imposed shear. The maximum deformation increases linearly with $\mathrm{Ca}$ when $\mathrm{Ca}<0.1$ and more slowly as $\mathrm{Ca}>0.2$. It reaches an asymptotic value when $\mathrm{Ca}$ is above a critical threshold $\mathrm{Ca} \approx 0.6$; in this regime the deformation is limited by the time over which constant shear is applied and not by the membrane deformability. The peak deformation is found to scale with the capsule-wall distance $\left(h_{\text {ini }} / a\right)^{1 / 3}$, as observed for capsules and droplets in near-wall steady shear $\underline{\underline{41}}$. We also discuss the phase delay between the capsule deformation and the background shear, another viscoelastic feature of the capsule dynamics.

The non-monotonic dependence of the capsule migration velocity on its deformability could be potentially used to sort cells. Oscillating a suspension in a Couette device, cells with the capillary number exhibiting the largest lateral migration velocity may be extracted and isolated. By tuning the oscillation frequency, specific cells may therefore be targeted. The role of the oscillations on the rheological behavior of a suspension is a challenging extension of the present work.

\section{ACKNOWLEDGEMENTS}

Computer time provided by SNIC (Swedish National Infrastructure for Computing) is gratefully acknowledged. We thank the anonymous referees for their inspiring comments. Lailai Zhu acknowledges the financial support from the European Research Council grant (ERC simcomics-280117) as a researcher at EPFL. Jean Rabault acknowledges the financial support received as a master student from École Polytechnique. Part of this work was supported by the Linné FLOW Centre at KTH and by the European Research Council Grant No. ERC-2013-CoG-616186, TRITOS to Luca Brandt.

\section{REFERENCES}

${ }^{1}$ T. Fischer and H. Schmid-Schönbein. Tank tread motion of red cell membranes in viscometric flow: behavior of intracellular and extracellular markers (with film). In Red Cell Rheology, pages 347-361. Springer, 1978. 
${ }^{2}$ T. Fischer, M. Stohr-Lissen, and H. Schmid-Schonbein. The red cell as a fluid droplet: tank tread-like motion of the human erythrocyte membrane in shear flow. Science, 202(4370):894-896, 1978.

${ }^{3}$ D. Barthès-Biesel. Motion of a spherical microcapsule freely suspended in a linear shear flow. J. Fluid Mech., 100(04):831-853, 1980.

${ }^{4}$ C. D. Eggleton and A. S. Popel. Large deformation of red blood cell ghosts in a simple shear flow. Phys. Fluids, 10:1834, 1998.

${ }^{5}$ M. Kraus, W. Wintz, U. Seifert, and R. Lipowsky. Fluid vesicles in shear flow. Phys. Rev. Lett., 77(17):3685, 1996.

${ }^{6} \mathrm{~J}$. Ha and S. Yang. Electrohydrodynamic effects on the deformation and orientation of a liquid capsule in a linear flow. Phys. Fluids, 12:1671, 2000.

${ }^{7}$ Y. Sui, H. T. Low, Y. T. Chew, and P. Roy. Tank-treading, swinging, and tumbling of liquid-filled elastic capsules in shear flow. Phys. Rev. E, 77(1):016310, 2008.

${ }^{8}$ E. Lac, D. Barthès-Biesel, N. A. Pelekasis, and J. Tsamopoulos. Spherical capsules in three-dimensional unbounded stokes flows: effect of the membrane constitutive law and onset of buckling. J. Fluid Mech., 516:303-334, 2004.

${ }^{9}$ D. V. Le. Effect of bending stiffness on the deformation of liquid capsules enclosed by thin shells in shear flow. Phys. Rev. E, 82(1):016318, 2010.

${ }^{10}$ V. V. Lebedev, K. S. Turitsyn, and S. S. Vergeles. Dynamics of nearly spherical vesicles in an external flow. Phys. Rev. Lett., 99:218101, Nov 2007.

${ }^{11}$ G. Ma, J. Hua, and H. Li. Numerical modeling of the behavior of an elastic capsule in a microchannel flow: The initial motion. Phys. Rev. E, 79:046710, Apr 2009.

${ }^{12}$ D. Cordasco and P. Bagchi. Orbital drift of capsules and red blood cells in shear flow. Phys. Fluids, 25:091902, 2013.

${ }^{13}$ T. Omori, Y. Imai, T. Yamaguchi, and T. Ishikawa. Reorientation of a nonspherical capsule in creeping shear flow. Phys. Rev. Lett., 108(13):138102, 2012.

${ }^{14}$ H. Zhao, E. SG. Shaqfeh, and V. Narsimhan. Shear-induced particle migration and margination in a cellular suspension. Phys. Fluids, 24:011902, 2012.

${ }^{15}$ I. Koleva and H. Rehage. Deformation and orientation dynamics of polysiloxane microcapsules in linear shear flow. Soft Matter, 8(13):3681-3693, 2012.

${ }^{16}$ Z. Peng and Q. Zhu. Deformation of the erythrocyte cytoskeleton in tank treading motions. Soft Matter, 9(31):7617-7627, 2013.

${ }^{17}$ D. Barthès-Biesel. The time-dependent deformation of a capsule freely suspended in a linear shear flow. J. Fluid Mech, 113:251-267, 1981. 
${ }^{18} \mathrm{C}$. Pozrikidis. Finite deformation of liquid capsules enclosed by elastic membranes in simple shear flow. J. Fluid Mech., 297:123-152, 1995.

${ }^{19}$ R. Finken, S. Kessler, and U. Seifert. Micro-capsules in shear flow. J. Phys-Condens. Mat., 23(18):184113, 2011.

${ }^{20} \mathrm{~S}$. R. Keller and R. Skalak. Motion of a tank-treading ellipsoidal particle in a shear flow. J. Fluid Mech., 120:27-47, 1982.

${ }^{21}$ J. M. Skotheim and T. W. Secomb. Red blood cells and other nonspherical capsules in shear flow: oscillatory dynamics and the tank-treading-to-tumbling transition. Phys. Rev. Lett., 98(7):078301, 2007.

${ }^{22}$ M. Abkarian, M. Faivre, and A. Viallat. Swinging of red blood cells under shear flow. Phys. Rev. Lett., 98(18):188302, 2007.

${ }^{23}$ T. Nakajima, K. Kon, N. Maeda, K. Tsunekawa, and T. Shiga. Deformation response of red blood cells in oscillatory shear flow. American Journal of Physiology-Heart and Circulatory Physiology, 259(4):H1071-H1078, 1990.

${ }^{24}$ S. Kessler, R. Finken, and U. Seifert. Elastic capsules in shear flow: Analytical solutions for constant and time-dependent shear rates. Eur. Phys. J. E Soft Matter, 29(4):399-413, 2009.

${ }^{25}$ J. Dupire, M. Abkarian, and A. Viallat. Chaotic dynamics of red blood cells in a sinusoidal flow. Phys. Rev. Lett., 104(16):168101, 2010.

${ }^{26}$ H. Noguchi. Dynamic modes of red blood cells in oscillatory shear flow. Phys. Rev. E, 81(6):061920, 2010.

${ }^{27}$ M. Zhao and P. Bagchi. Dynamics of microcapsules in oscillating shear flow. Phys. Fluids, 23(11):111901-111901, 2011.

${ }^{28}$ R. Haddock and C. Hill. Rhythmicity in arterial smooth muscle. The Journal of physiology, 566(3):645-656, 2005.

${ }^{29}$ K. Shimamura, F. Sekiguchi, and S. Sunano. Tension oscillation in arteries and its abnormality in hypertensive animals. Clinical and experimental pharmacology and physiology, 26(4):275-284, 1999.

${ }^{30}$ H. Nilsson and C. Aalkjær. Vasomotion: mechanisms and physiological importance. Molecular interventions, 3(2):79, 2003.

${ }^{31}$ C. Aalkjaer and H. Nilsson. Vasomotion: cellular background for the oscillator and for the synchronization of smooth muscle cells. British journal of pharmacology, 144(5):605-616, 2005. 
${ }^{32}$ C. Meyer, G. De Vries, ST. Davidge, and DC. Mayes. Reassessing the mathematical modeling of the contribution of vasomotion to vascular resistance. J. Appl Physiol., 92(2):888$889,2002$.

${ }^{33}$ TW. Secomb, M. Intaglietta, and JF. Gross. Effects of vasomotion on microcirculatory mass transport. Prog Appl Microcirc, 15:49-61, 1989.

${ }^{34}$ Daniel D. Goldman and A. Popel. A computational study of the effect of vasomotion on oxygen transport from capillary networks. J. Theor. Biol., 209(2):189-199, 2001.

${ }^{35}$ R. Fåhræus and T. Lindqvist. The viscosity of the blood in narrow capillary tubes. American Journal of Physiology-Legacy Content, 96(3):562-568, 1931.

${ }^{36} \mathrm{~S}$. K. Doddi and P. Bagchi. Lateral migration of a capsule in a plane Poiseuille flow in a channel. Int. J. Multiphas. Flow., 34(10):966-986, October 2008.

${ }^{37}$ P. Pranay, R. G. Henríquez-Rivera, and M. D. Graham. Depletion layer formation in suspensions of elastic capsules in newtonian and viscoelastic fluids. Phys. Fluids, 24:061902, 2012 .

${ }^{38}$ B. Kaoui, GH. Ristow, I. Cantat, C. Misbah, and W. Zimmermann. Lateral migration of a two-dimensional vesicle in unbounded poiseuille flow. Phys. Rev. E, 77(2):021903, 2008.

${ }^{39}$ G. Danker, P. M. Vlahovska, and C. Misbah. Vesicles in poiseuille flow. Phys. Rev. Lett., 102(14):148102, 2009.

${ }^{40}$ H. Zhao, AP. Spann, and ES. Shaqfeh. The dynamics of a vesicle in a wall-bound shear flow. Phys. Fluids, 23(12):121901, 2011.

${ }^{41}$ RK. Singh, X. Li, and K. Sarkar. Lateral migration of a capsule in plane shear near a wall. J. Fluid Mech., 739:421-443, 2014.

${ }^{42}$ S. Nix, Y. Imai, D. Matsunaga, T. Yamaguchi, and T. Ishikawa. Lateral migration of a spherical capsule near a plane wall in stokes flow. Phys. Rev. E, 90(4):043009, 2014.

${ }^{43} \mathrm{D}$ Matsunaga, Y Imai, T Yamaguchi, and T Ishikawa. Deformation of a spherical capsule under oscillating shear flow. J. Fluid Mech., 762:288-301, 2015.

${ }^{44}$ D. Barthès-Biesel, J. Walter, and A.-V. Salsac. Flow-induced deformation,of artificial capsules. In C. Pozrikidis, editor, Computational hydrodynamics of capsules and biological cells. CRC Press, 2010.

${ }^{45}$ J. P. Hernández-Ortiz, J. J. de Pablo, and M. D. Graham. Fast computation of manyparticle hydrodynamic and electrostatic interactions in a confined geometry. Phys. Rev. Lett., 98(14):140602, 2007.

${ }^{46}$ P. F. Fischer, J. W. Lottes, and S. G. Kerkemeier. nek5000 Web page, 2008. http://nek5000.mcs.anl.gov. 
${ }^{47}$ H. Zhao, A. H. G. Isfahani, L. N. Olson, and J. B. Freund. A spectral boundary integral method for flowing blood cells. J. Comput. Phys., 229:3726-3744, 2010.

${ }^{48}$ Q. Huang and T. A. Cruse. Some notes on singular integral techniques in boundary element analysis. Int. J. Numer. Meth. Eng, 36:2643-2659, 1993.

${ }^{49}$ L. Zhu, E. Lauga, and L. Brandt. Low-reynolds number swimming in a capillary tube. $J$. Fluid Mech., 726:285-311, 2013.

${ }^{50} \mathrm{~L}$. Zhu and L. Brandt. The motion of a deforming capsule through a corner. J. Fluid Mech., 770:374-397, 2015.

${ }^{51}$ L. Zhu. Simulation of individual cells in flow. Phd dissertation, Royal Institute of Technology, Stockholm, Mar 2014.

${ }^{52}$ L. Zhu, C. Rorai, M. Dhrubaditya, and L. Brandt. A microfluidic device to sort capsules by deformability: a numerical study. Soft Matter, 10:7705-7711, 2014.

${ }^{53}$ C. Rorai, A. Touchard, L. Zhu, and L. Brandt. Motion of an elastic capsule in a constricted microchannel. Eur. Phys. J. E Soft Matter, 38(5):1-13, 2015.

${ }^{54}$ G. d'Avino, PL. Maffettone, F. Greco, and MA. Hulsen. Viscoelasticity-induced migration of a rigid sphere in confined shear flow. J. Non-Newtonian Fluid Mech., 165(9):466-474, 2010 .

${ }^{55}$ E. Lauga and T.R. Powers. The hydrodynamics of swimming microorganisms. Rep. Prog. Phys., 72:096601, 2009.

${ }^{56}$ O. S. Pak, T. Normand, and E. Lauga. Pumping by flapping in a viscoelastic fluid. Phys. Rev. E, 81:036312, 2010.

${ }^{57}$ NC. Keim, M. Garcia, and PE. Arratia. Fluid elasticity can enable propulsion at low reynolds number. Phys. Fluids, 24(8):081703, 2012.

${ }^{58}$ Tian Qiu, Tung-Chun Lee, Andrew G. Mark, Konstantin I. Morozov, Raphael Münster, Otto Mierka, Stefan Turek, Alexander M. Leshansky, and Peer Fischer. Swimming by reciprocal motion at low reynolds number. Nat Commun, 5, 112014.

${ }^{59}$ E. M. Purcell. Life at low Reynolds number. Am. J. Phys., 45:3-11, 1977.

${ }^{60} \mathrm{H}$. Ma MD. Graham. Theory of shear-induced migration in dilute polymer solutions near solid boundaries. Phys. Fluids, 17(8):083103, 2005.

${ }^{61}$ B. Liu, T. R. Powers, and K. S. Breuer. Force-free swimming of a model helical flagellum in viscoelastic fluids. Proc. Natl. Acad. Sci. USA, 108:19516-19520, 2011.

${ }^{62}$ SE. Spagnolie, B. Liu, and TR. Powers. Locomotion of helical bodies in viscoelastic fluids: enhanced swimming at large helical amplitudes. Phys. Rev. Lett., 111(6):068101, 2013. 
${ }^{63} \mathrm{C} . \mathrm{H}$. Wiggins and R. E. Goldstein. Flexive and propulsive dynamics of elastica at low reynolds number. Phys. Rev. Lett., 80(17):3879, 1998.

${ }^{64}$ S. Y. Tony, E. Lauga, and AE. Hosoi. Experimental investigations of elastic tail propulsion at low reynolds number. Phys. Fluids, 18(9):091701, 2006.

${ }^{65}$ R. M. Arco, J. R. Vélez-Cordero, E. Lauga, and R. Zenit. Viscous pumping inspired by flexible propulsion. Bioinspiration $\mathscr{G}$ biomimetics, 9(3):036007, 2014.

${ }^{66}$ KE. Machin. Wave propagation along flagella. J. exp. Biol, 35(4):796-806, 1958.

${ }^{67}$ H. M. Shapiro. Practical flow cytometry. John Wiley \& Sons, 2005.

${ }^{68}$ S. Ye, X. Shao, Z. Yu, and W. Yu. Effects of the particle deformability on the critical separation diameter in the deterministic lateral displacement device. J. Fluid Mech., 743:60-74, 2014.

${ }^{69}$ T. Krueger, D. Holmes, and P. V. Coveney. Deformability-based red blood cell separation in deterministic lateral displacement devicesa simulation study. Biomicrofluidics, $8(5): 054114,2014$.

${ }^{70} \mathrm{X}$. Li and K. Sarkar. Front tracking simulation of deformation and buckling instability of a liquid capsule enclosed by an elastic membrane. J. Comput. Phys., 227(10):4998-5018, 2008.

${ }^{71} \mathrm{PC}-\mathrm{H}$. Chan and LG. Leal. The motion of a deformable drop in a second-order fluid. $J$. Fluid Mech., 92(01):131-170, 1979.

${ }^{72} \mathrm{M}$. Shapira and S. Haber. Low reynolds number motion of a droplet in shear flow including wall effects. Int. J. Multiphase Flow, 16(2):305-321, 1990.

${ }^{73}$ G. Ghigliotti, A. Rahimian, G. Biros, and C. Misbah. Vesicle migration and spatial organization driven by flow line curvature. Phys. Rev. Lett., 106(2):028101, 2011. 


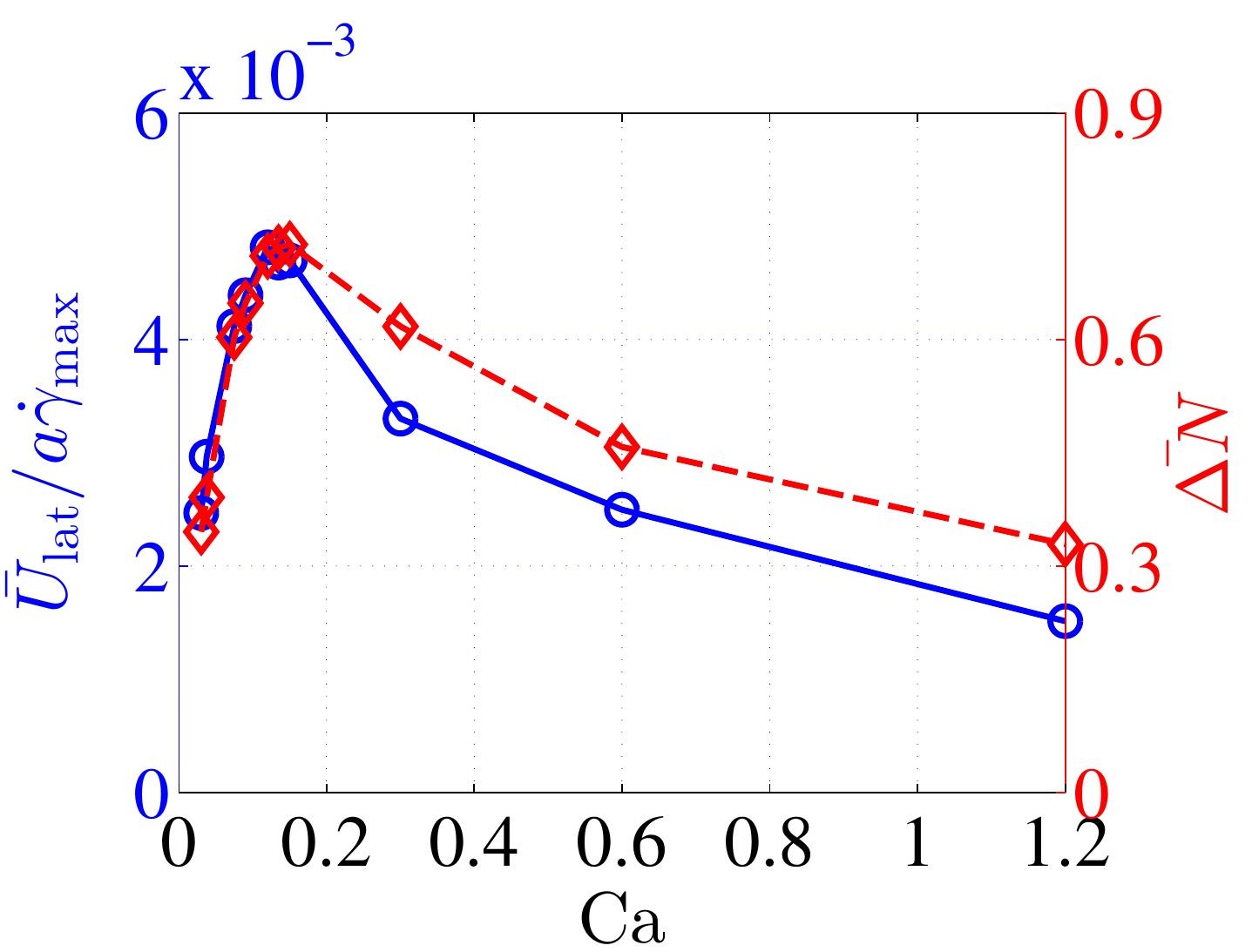

